\title{
Processing the Couette viscometry data using a Bingham approximation in shear rate calculation
}

\author{
Patrice Estellé $^{1, *}$, Christophe Lanos ${ }^{1}$, Arnaud Perrot ${ }^{2}$ \\ ${ }^{1}$ LGCGM, Département Matériaux et Thermo-Rhéologie, \\ Institut Universitaire Technologique, rue du Clos Courtel, BP 90422, \\ 35704 Rennes Cedex 7, France \\ ${ }^{2}$ LG2M, Université de Bretagne Sud, \\ Rue Saint Maudé, 56321 Lorient Cedex, France
}

${ }^{*}$ Author to whom correspondence should be addressed.
Electronic mail: patrice.estelle@ univ-rennes1.fr

Tel: +33 (0) 23234200

Fax: +33 (0) 223234051

\begin{abstract}
:
This paper presents an approach to computing the shear flow curve from torque-rotational velocity data in a Couette rheometer. The approximation techniques in shear rate calculation are generally dictated by the radius ratio between coaxial cylinders and the rheological behaviour of fluid tested. Here, the approach consists in analysing the sheared material as a Bingham fluid and computing an average shear rate when the fluid in the cylindrical gap is partially and fully sheared. We focus in particular on the applicability of the Bingham approximation in shear rate calculation. First, the approach is assessed by examining synthetic data generated with Newtonian, non-Newtonian and yield stress materials with known properties, varying the gap radius ratio. The results, which are compared with commonly used techniques in shear rate calculation, prove the relevance of the proposed approach. Then, its efficiency is examined by applying it to process Couette data of yield stress fluids taken from published works.
\end{abstract}

Keywords: shear flow, Couette viscometer, rheometry, Bingham model

\section{Introduction}

The Searle-Couette system or rotational coaxial cylinder viscometer is widely used for rheological measurements. It consists of two coaxial cylinders with a fluid being placed in the 
annulus between them. The torque and rotational velocity of the inner cylinder are measured to determine shear stress and shear rate. The shear stress can be directly calculated from the measured torque. However, the problem involved in determining the shear rate is not as straightforward and has been the subject of intensive research for a long time. Actually, in such a geometry, the shear stress and shear rate are not uniformly distributed within the gap [1]. Moreover, correct evaluation of shear rate distribution is not independent of the fluid type and requires an a priori assumption of a fluid model. It also depends on the gap size between the cylinders. So, numerous methods or solutions have been proposed for the determination of shear rate value. The first attempt in evaluating the shear rate is attributed to Mooney [2] who considered the shear rate to be homogeneous within the gap. He also assumed the shear stress to be the arithmetic mean of the stresses in the bob and cup surfaces. Later, to correct the homogeneous approximation, Moore and Davies [3] derived the infinite series for the shear rate corresponding to the geometric means of the shear stresses at the bob and cup surfaces. Finally, Krieger and Elrod [4] derived an infinite series for the shear rate at the bob that can be arranged in closed form so that the dominant term is identical to the power-law behaviour [5]. Yang and Krieger [6] also used a series solution procedure to a model fluid with yield stress. Darby [7] and Nguyen and Boger [1] have shown that Krieger's approach did not correctly predict the shear rate at the bob of yield stress fluids in particular when the fluid is partially sheared within the gap. Krieger's approach is also limited for noised experimental data [8]. Recently, Yeow and co-workers [9-11] have proposed the Tikhonov regularization method to evaluate the Couette shear flow curve of various materials. The main advantage of this method is two-fold: it requires neither gap approximation nor prespecification of rheological constitutive equation. However, the Tikhonov regularization method depends on the proper choice of a regularization parameter and induces iterative procedure to obtain flow curve and yield stress value when it exists. More recently, Ancey [12] has reviewed the socalled Couette inverse problem and has used wavelet-vaguelette decomposition to recover the shear rate at the bob. This approach has been shown to be particularly appropriate for complex fluids such as yield stress material and granular suspension. With such an approach, rheological behaviour and gap approximation are not needed. Nevertheless, the procedure first requires the filtering of the data. Lately, de Hoog and Anderssen [13] derived the flow curve of Couette rheometer data from the Euler-Maclaurin sum formula solution of the equation given by Krieger and Elrod [4]. The performance of this formula has been numerically analysed and demonstrated from non-Newtonian and yield stress material Couette data under narrow gap limitation. 
As previously proposed, the purpose of the present work is to develop a method for analysing the Couette viscometer data. The approach considered consists in approximating the sheared material as a Bingham fluid and computing an average shear rate at the bob when the annulus is partially and/or fully sheared, as detailed in section 2. The applicability and the performance of the proposed approximation technique in shear rate calculation is given and analysed numerically in section 3, considering Newtonian, non-Newtonian and yield stress materials and various annular gaps. The proposed approach has also been shown to allow appropriated flow curves to be recovered. Then, the Bingham approximation is examined for noisy ideal Couette rheometer data. It is finally applied to published Couette experimental data of yield stress materials in section 4 .

\section{Shear rate approximation technique}

We consider the Couette geometry of an inner cylinder, called the bob, of length $\mathrm{h}$ and radius $R_{b}$, and an outer cylinder, called the cup, of radius $R_{c} . \Omega$ is the rotational velocity of the inner cylinder. We assume that the inner cylinder rotates, the outer cylinder is stationary and a noslip boundary condition is achieved between the inner cylinder and the sheared fluid. The fluid is also assumed to be incompressible and inelastic, edge and inertia effects as well as shearing effect in the bottom zone are neglected.

As previously mentioned, the shear stress exerted on the inner cylinder can be directly related to the torque as follows:

$$
\tau_{b}(M)=\frac{M}{2 \pi h R_{b}^{2}}
$$

The shear stress at the bob is independent of the nature of the fluid. The angular velocity can be described in terms of shear stress and shear rate as follows:

$$
\Omega=\int_{R_{b}}^{R_{c}} \frac{\dot{\gamma}(r)}{r} d r=\frac{1}{2} \int_{\tau_{b}}^{\tau_{c}} \frac{\dot{\gamma}(\tau)}{\tau} d \tau .
$$

Several techniques allow equation (2) to be solved. The commonly used approximations are simple shear approximation, Newtonian approximation, Power-law approximation and 
Krieger method. These expressions are presently included for comparison purposes with the analysis developed in this paper.

With a very narrow gap $\left(R_{c}-R_{b} \leq R_{b}\right)$, the system approaches simple shear Couette flow. Assuming a uniform shear rate across the gap gives [14]

$$
\dot{\gamma}=\frac{\Omega R_{b}}{R_{c}-R_{b}}=\frac{\Omega}{\alpha-1} .
$$

Where, $\alpha$ is the ratio of cup radius, $R_{c}$ to bob radius $R_{b}$, and $\Omega$ is the angular velocity ( $\left.\mathrm{rad} / \mathrm{s}\right)$. For the gap to be classed as narrow, $1 / \alpha$ must be greater than 0.97 [15]. When calculating shear rates with this approximation, corresponding average shear stress proposed by Mooney [2] should be used.

$$
\tau_{a}=\frac{1}{2}\left(\tau_{c}+\tau_{b}\right)=\frac{M\left(1+\alpha^{2}\right)}{4 \pi h R_{c}{ }^{2}}
$$

Where $\tau_{a}$ is the average shear stress, $\tau_{c}$ is the shear stress at the cup, and $\tau_{b}$ is the shear stress at the bob.

For Newtonian fluids, the shear rate at the bob in a concentric geometry can be calculated from the following expression $[14,16]$,

$$
\dot{\gamma}=2 \Omega\left(\frac{\alpha^{2}}{\alpha^{2}-1}\right) .
$$

Commercial viscometers frequently use equation (5) to approximate the shear rate. Newtonian shear rates need to be corrected for non-Newtonian behaviour with the correction term depending on the extent of the deviation from Newtonian behaviour. The shear rate factors may differ according to the equations used and the size of the gap, as respectively shown by Martinez-Padilla and Rivera-Vargas [17] and Joye [18]. In order to minimize errors in calculated shear rates it is advisable to work with concentric cylinders which have a narrow gap. Yet, the difficulty of coping with suspensions containing large particles is an important limitation of narrow gap approximation. As a consequence, a wide gap is usually preferred to study yield stress materials. With such materials, a plug flow region may appear within the gap as shown by [1] who have also proposed using the following expression: 


$$
\dot{\gamma}=2 M \frac{d \ln \Omega}{d \ln M} .
$$

Equation (6) is the exact result for a bob rotating in unbounded fluid.

For a power-law fluid, the shear rate at the bob can be derived [14] as,

$$
\dot{\gamma}=\frac{2 \Omega}{n} \frac{\alpha^{2 / n}}{\alpha^{2 / n}-1} .
$$

Where, $\mathrm{n}$ is the consistency coefficient. The flow behaviour index is calculated by evaluating the derivative of $\ln \tau_{\mathrm{b}}$ with respect to $\ln \Omega$ [14]. In order to correct the non-Newtonian behaviour of power-law fluids in concentric cylinder geometry, an expression presented by Krieger and Elrod [4] has been extensively used

$$
\dot{\gamma}=\frac{\Omega}{\ln \alpha}\left(1+\ln \alpha \frac{d \ln \Omega}{d \ln \tau}+\frac{(\ln \alpha)^{2} d^{2} \Omega}{3 \Omega d(\ln \tau)^{2}}\right) .
$$

The Krieger solution is very close to power-law approximation. So, when the flow behaviour index, $\mathrm{n}$, can be calculated at each $\tau_{\mathrm{b}}$, the power-law approximation solution provides excellent approximation for the shear rate at the bob.

In the present work, the rheological behaviour of sheared fluid is modelled by the Bingham constitutive equation, which relates the shear stress $\tau$ and the shear rate $\dot{\gamma}$ by the following relationships:

$$
\tau=K+\mu \dot{\gamma}, \text { if } \tau>\mathrm{K} .
$$

Where, $\mathrm{K}$ is the yield stress and $\mu$ is the plastic viscosity.

For a Bingham plastic model, two distinct conditions may occur in the annulus: (1) $\tau_{c} \leq K \leq \tau_{b}$; (2) $\tau_{c}>K$. The first condition implies that a rigid plug flow occurs in the region near the cup, while the second one indicates that the yield value is exceeded in the entire annulus and the fluid within the gap is fully sheared. Therefore, the rotational velocity has a separate solution corresponding to each of these two solutions. The rotational velocity 
can also be described in terms of torque, Bingham model parameters and Couette geometrical system as follows:

$$
\begin{gathered}
\Omega=\frac{K}{2 \mu}\left[\ln \left(2 \pi h K R_{b}^{2}\right)-1\right]+\frac{M}{4 \pi h \mu R_{b}{ }^{2}}-\frac{K}{2 \mu} \ln (M), \text { if } \tau_{c} \leq K \leq \tau_{b}, \text { (10) } \\
\Omega=\frac{M}{4 \pi h \mu}\left(\frac{1}{R_{b}{ }^{2}}-\frac{1}{R_{c}{ }^{2}}\right)+\frac{K}{\mu} \ln \left(\frac{R_{b}}{R_{c}}\right), \text { if } \tau_{c}>K
\end{gathered}
$$

Equation (11) corresponds to the Reiner-Rivlin expression.

The derivative of equations (10) and (11) with respect to the torque yields

$$
\begin{aligned}
& \frac{d \Omega}{d M}=\frac{1}{4 \pi h \mu R_{b}^{2}}-\frac{K}{2 \mu M}, \text { if } \tau_{c} \leq K \leq \tau_{b}, \\
& \frac{d \Omega}{d M}=\frac{1}{4 \pi h \mu}\left(\frac{1}{R_{b}^{2}}-\frac{1}{R_{c}^{2}}\right), \text { if } \tau_{c}>K .
\end{aligned}
$$

The shear rate for the Bingham plastic model can be expressed in terms of rotational velocity, torque, radius of cylinders by combining equations (1), (9), (12) and (13), as follows:

$$
\begin{aligned}
& \dot{\gamma}=2 M \frac{d \Omega}{d M}, \text { if } \tau_{c} \leq K \leq \tau_{b}, \\
& \dot{\gamma}=2 \frac{M \frac{d \Omega}{d M}}{\left(1-\frac{R_{b}{ }^{2}}{R_{c}{ }^{2}}\right)}-\frac{\Omega-M \frac{d \Omega}{d M}}{\ln \left(\frac{R_{b}}{R_{c}}\right)}, \text { if } \tau_{c}>K .
\end{aligned}
$$

The first equation of shear rate corresponds to the solution developed by [1], see equation (6), for a yield stress material partially sheared within the gap. It can be noted that equation (14) does not depend on the rheological model of the yield stress fluid considered. The second equation is used to recover the shear rate for non yield stress materials or for completely sheared condition. So, as proposed in [1], the shear rate is a combination of two expressions following the flow condition in the annulus. It is considered that the appropriate value of shear rate is one which maximizes the energy dissipation in the flowing sample. Then, the 
characteristic shear rate is defined as

$$
\dot{\gamma}=\max (\text { equation(14); equation(15)). }
$$

This does not require the knowledge of the fluid yield stress value a priori or the identification of the flow regime in the annulus.

It is assumed that a series of torque measurements $\mathrm{M}_{\mathrm{j}}$ (corresponding to stress $\tau_{j}$ ) are made at a series of increasing (or decreasing) rotation rate $\Omega_{j}$. The derivative $\frac{d \Omega}{d M}$ is approximated by $\frac{\Omega_{j}-\Omega_{j-1}}{M_{j}-M_{j-1}}$. Once the shear rate has been estimated by Eq. (16), it is deemed to correspond to the following wall shear stress

$$
\tau=\frac{1}{2}\left(\tau_{j}+\tau_{j-1}\right)
$$

It is worth noting that the proposed equations are constructed on the linear derivative of the angular velocity versus the applied (or measured) torque. Logarithmic derivatives are nevertheless more appropriate for logarithmic variation of the torque (or the velocity).

\section{Numerical results}

As was generally done in the works discussed in the introduction, comparison of the various shear rate approximations are investigated from representative fluids in the absence of noise. Presently, we have considered Newtonian, power law, Herschel-Bulkley and Casson fluids. Here, the goal is to show that the Bingham approximation allows for the appropriated flow curves of different fluids to be recovered, independently of fluid behaviour and gap size, and that the shear rate evaluation is better than one coming from approximations mentioned previously.

For the Newtonian and power law fluids, the shear rate is related to the shear stress by the following relationships.

$$
\begin{aligned}
& \tau=\mu \dot{\gamma}, \\
& \tau=\eta \dot{\gamma}^{n} .
\end{aligned}
$$

where $\mu$ is the viscosity, $\eta$ is the consistency and $n$ the flow behaviour index. 
The constitutive relationship for the generalized Herschel-Bulkley model has the following form:

$$
\tau=K+\eta \dot{\gamma}^{n}, \text { when } \tau>\mathrm{K}
$$

Where $\mathrm{K}$ is the yield stress.

As used in works concerning Couette rheometry [1, 9, 12, 13], we have also considered a Casson fluid. Its constitutive relationship is given by

$$
\sqrt{\tau}=\sqrt{K}+\sqrt{\mu \dot{\gamma}}, \text { when } \tau>\mathrm{K}
$$

In the current study, the rheological and Couette geometrical parameters used to generate torque simulated data are described in Table 1. The set of torque values are numerically obtained from equation (1). For Newtonian and power law fluids, the rotation rate relation can be obtained analytically via respectively equation (5) and (7). The rotation rate of HerschelBulkley and Casson fluids is obtained from the finite difference method with $\Delta \mathrm{r}=\left(\mathrm{R}_{\mathrm{c}^{-}}\right.$ $\mathrm{R}_{\mathrm{b}}$ )/100. We have considered three different radius ratios, 1.04, 2 and 3, corresponding respectively to narrow, moderate and large gaps in Couette rheometer. As done in [1], the performance of our approach compared to other approximations is estimated by computing the percentage deviation of the calculated shear rate from the true shear rate at the bob which was taken from the fluid model assumed.

\subsection{Newtonian and power law fluids}

The errors in the shear rate of Newtonian fluid are independent of shear stress for all the radius ratios envisaged, respectively 1.04, 2 and 3. As expected, the Newtonian approximation provides an accurate estimation of shear rate at any given shear stress. A similar result is obtained from the Bingham approximation technique, as the calculated errors are less than $10^{-}$

${ }^{11} \%$ for the radius ratios used. The error involved in using simple shear approximation appears to be large, $5.7 \%$ with narrow gap and increases to $62.5 \%$, respectively $77 \%$ with increasing radius ratio to 2 , respectively 3 . It is worth noting that it was checked that the shear rate of eq.(15) is always greater than the one obtained with eq.(14), as expected, due to the viscous behaviour of the fluid investigated here.

The power law fluid represents a more general test than the Newtonian fluid. Figure 1 compares the calculated errors in the shear rate as a function of shear stress for similar radius 
ratios, respectively 1.04, 2 and 3, using our approximation, the Newtonian, the power law and the simple shear approximations. A comparison of plots in Figure 1 shows that the simple shear and Newtonian approximations perform poorly. As previously mentioned, the error involved increases with increasing radius ratio. It tends to $92.5 \%$, respectively $66.3 \%$, for the large gap. As expected, the power law approximation provides the exact solution in calculated shear rate for the three different radius ratios. As further shown in Figure 1, the performance of the Bingham approximation is also quite accurate for any radius ratio. It provides a suitable value of shear rate. In the region up to a shear stress of $50 \mathrm{~Pa}$, the error is respectively $0.35 \%$ for narrow gap, $1 \%$ for moderate gap and the error ranges 0.5 to $0.07 \%$ as the shear stress increases for large gap. Here again, it was checked that the shear rate of eq.(15) is always greater than the one obtained with eq.(14).

\subsection{Yield stress fluids}

Figure 2 shows that the shear rate of the Herschel-Bulkley fluid is accurately determined by the solution of [1] when the fluid is partially sheared, as would be expected. It also shows the transition between both solutions which is presently used to compute the shear rate when the yield stress is achieved at the cup and when the gap becomes fully sheared. This provides a first idea of the flow regime in the annulus. When the annulus is fully sheared, results in Figure 2 demonstrate that commonly used approximations are no longer applicable, whereas the Bingham approximation provides accurate determination of the shear rate.

When the annulus is fully sheared, as done with both narrow and moderate gaps, results in Figure 3 show that the Bingham approximation provides the best determination of the shear rate of the Casson fluid. When a plug flow region is present in the annulus, the shear rate here is also correctly determined by the solution of [1]. Figure 3 also clearly shows the transition between both solutions and allows the flow regime transition in the annulus to be detected. As previously shown, the use of Newtonian approximation leads to substantial errors in calculated shear rate of yield stress materials even for fully sheared flow condition.

\subsection{Influence of experimental noise}

In order to evaluate the effects of experimental noise on the performance of the Bingham approximation technique in shear rate calculation, we have considered the Couette noisecorrupted synthetic data of Casson fluid used by [9]. These authors have evaluated the 
performance of the Thikonov regularisation from the Casson model fluid previously used in [1] adding Gaussian random noise with zero mean and standard deviation of $10 \%$ to the rotational speed obtained from the Casson constitutive equation. The first set of these simulated noisy data, which are detailed in Table 1(a) of [9] and presently used, corresponds to a radius ratio of 1.1. As mentioned in [9], it can be noted that the fluid is completely sheared with the narrow gap (radius ratio of 1.1). The data corresponding to the moderate gap (radius ratio of 2) are not used, because in this case, the fluid is partially sheared [9] and the solution of Nguyen and Boger [1] is sufficient to evaluate the shear rate. The values of wall shear stress are then used to determine the corresponding torque from equation (1) and the Couette geometry system. For comparison purpose, we have considered the arithmetic mean of the wall shear stress and shear rate at the bob obtained from synthetic data. As a result, one can see in Figure 4 that the Bingham approximation accurately determines the shear rate at the bob for the completely sheared condition. It also shows that the calculated Couette shear flow curve of noisy ideal Casson fluid appears to correspond with the theoretical shear flow curve. The average percentage deviation between the computed curve from the Bingham approximation and the synthetic data is $5.3 \%$. This is of the same order of magnitude as the random noise added and the average deviation obtained from the Thikonov regularisation [9].

To improve the resulting shear flow curve, it should be necessary to first smooth the data [12] before applying the Bingham approximation technique or use a centered difference for the calculation of shear rate.

\section{Experimental application}

\subsection{Tomato ketchup}

Steffe [14] has published a set of Couette viscometry data for commercial tomato ketchup, which has been used by Leong and Yeow [11] for a practical application of the Tikhonov regularization. The dimensions of the Couette system used for the measurement are detailed in [14] and the outer/inner radius ratio is 1.048 . So, Steffe [14] has treated experimental data using narrow gap approximation and also describing the tomato ketchup as a Power Law fluid. The shear rate vs shear stress relationships obtained by Steffe [14] is described in Fig. 5 . The shear stress range covered by his data is between $22.85 \mathrm{~Pa}$ and $130.12 \mathrm{~Pa}$. The lower shear stress value obtained by Leong and Yeow [11] is $20.81 \mathrm{~Pa}$ while the higher shear stress value is the one obtained by Steffe [14]. The shear rate-shear stress curve obtained from the Bingham approximation, without first smoothing the data, is shown in Fig. 5. Good 
correlation is achieved between the data of Steffe [14] and the present work, as also obtained by [11] from the Tikhonov regularization. Because two rotational velocity $\Omega$ and torque $M$ values are used to iteratively process the Couette viscometry data, the shear stress range covered by our analysis is not as large as the one covered by Steffe [14] and Leong and Yeow [11]. It varies between 24.57 Pa and 113.28 Pa. Furthermore, Figure 5 shows that Newtonian approximation performs poorly compared to other techniques while the gap is narrow, in particular at high shear rate.

While Steffe [14] has used a power law model to represent the tomato ketchup flow curve, Leong and Yeow [11] have shown that this material has a yield stress equal to $18.45 \mathrm{~Pa}$. We have modelled the shear flow curve obtained from the Bingham approximation with the general Herschel-Bulkley constitutive equation. The yield stress value obtained is also 18.47Pa, as the flow index and consistency are the same than ones determined by Steffe [14]. This yield stress value is in accordance with the results of Leong and Yeow [11].

\subsection{Bentonite suspensions}

Kelessidis and Maglione [19] have reported Couette viscometry data of aqueous bentonite suspensions. This is reproduced in Table 2 for two yield stress suspensions, respectively W2 ( $5 \mathrm{wt} . \%$ bentonite suspension) and $\mathrm{W} 4$ ( $6.42 \mathrm{wt} . \%$ bentonite suspension). These authors have evaluated in particular the shear rate and rheological parameters of the suspensions from both Casson and Robertson-Stiff constitutive equations, which have also been compared to Newtonian approximation results. The rotational viscometer used in this work has a stationary inner cylinder of $0.017245 \mathrm{~mm}$ in radius and $0.038 \mathrm{~mm}$ in height. The radius of the rotating outer cup is $0.018415 \mathrm{~mm}$. Here the radius ratio is 1.0678 . Mixing of suspensions and experimental procedure is described in [19]. Following the steps previously mentioned, the Bingham approximation is then applied to process directly the data in Table 2. The resulting shear rate-shear stress curves are given in Figure 6 for both suspensions and compared respectively to the shear flow curve obtained by Kelessidis and Maglione [19] from Casson model and Newtonian approximation. Figure 6 shows that the Bingham approximation provides accurate determination of the Couette shear flow of bentonite suspensions. The average percentage deviation of the calculated shear rate from the shear rate obtained from Casson model is respectively less than $3 \%$ for both suspensions. Moreover, the Newtonian approximation is shown to lead to a large error in the calculated shear rate even for narrow gap and fully sheared flow, as predicted in [19]. It should be mentioned than the shear rate 
obtained from the Bingham approximation spans the range $80-8831 / \mathrm{s}$ for W2 and W4. This is smaller that the shear rate range of [19] due to the numerical differentiation used in the Bingham approximation.

\section{Concluding remarks}

In this paper we have proposed to evaluate the shear rate in Couette rheometer assuming the sheared material as a Bingham fluid. This provides separate shear rate solutions depending on the flow regime within the gap. Where the material is partially sheared, the shear rate is accurately determined by the solution of [1]. For completely sheared flow condition, the Bingham approximation presently developed provides a suitable value of the shear rate. The relevance of the processing Couette viscometry from the Bingham technique approximation has been investigated from both ideal and real data, also using different viscometer geometries. In the shear rate calculation, the Bingham approximation technique has proven to be a general and practical method for recovering the Couette data of Newtonian and nonNewtonian materials under various gap sizes. Here, the shear rate values obtained from the Bingham approximation technique appear to be better than commonly used approximation techniques in the shear rate calculation.

With yield stress materials, as the previous comparisons have shown, the presently used shear rate calculation overcomes the problem of a priori knowledge of the yield stress value nor the flow regime identification within the gap. It was found that, when fully sheared flow is achieved, the shear rate can be correctly computed using the Bingham approximation technique. For narrow and moderate gaps, it was shown that the transition between both solutions of shear rate allows the flow regime modification to be evaluated.

Shear rate calculation from Bingham approximation has been successfully compared to previous published Couette results of yield stress materials. The shear stress - shear rate range obtained in this work covers an appropriate domain, while the processing computes a number of discrete points rather than a continuous curve as was provided in [9,12]. The major advantage of our analysis is that it does not require narrow or wide gap approximation and shear factor calculations. It can be finally noted that the Tikhonov regularization method depends on the proper choice of a regularization parameter, for denoising the data. Here, the set of torque-rotational velocity data and Couette geometry system are only needed to process the shear stress-shear rate curve. 
Acknowledgement: The reviewers' comments that have contributed to make this article more complete are gratefully acknowledged.

\section{References}

[1] Nguyen Q.D., Boger D.V., Characterization of yield stress fluids with concentric cylinder viscometers Rheol. Acta 26 508-515 (1987)

[2] Mooney M., Explicit formulas for slip and fluidity, J. Rheology 2 210-222 (1931)

[3] Moore F., Davies L.J., The consistency of ceramics slip, Trans. Br. Cer. Soc. 55 313-338 (1956)

[4] Krieger I.M., Elrod H, Direct determination of the flow curves of non-Newtonian fluids. II Shearing rate in the concentric cylinder viscometer, J. Applied Physics 24(2) 134-136 (1953)

[5] Krieger I.M., Shear rate in the Couette viscometer, J. Rheology 12(1) 5-11 (1968)

[6] Yang T.M.T., Krieger I.M., Comparison of methods for calculating shear rates in coaxial viscometers, J. Rheology 22 413-421 (1978)

[7] Darby R., Couette viscometer data reduction for materials with a yield stress, J. Rheology 29 369-378 (1985)

[8] Borgia A., Spera F., Error analysis for reducing noisy wide-gap concentric cylinder rheometric data for nonlinear fluids: Theory and applications, J. Rheology 34 117-135 (1990)

[9] Yeow Y.L., Woan C.K., Pannie P.P.T., Solving the inverse problem of Couette viscometry by Tikhonov regularization, J. Rheology 44(6) 1335-1351 (2000)

[10] Yeow Y.L., Wickramasinghe S.R., Leong Y.K., Han B, Model-independent relationship between hematocrit, blood viscosity and yield stress derived from Couette viscometry data, Biotechnol. Prog. 18 1068-1075 (2002)

[11] Leong, Y.K., Yeow, Y.L., Obtaining the shear stress shear rate relationship and yield stress of liquid foods from Couette viscometry data, Rheologica Acta 42 365-371 (2003)

[12] Ancey C., Solving the Couette inverse problem using a wavelet-vaguelette decomposition, J. Rheology 49(2) 441-460 (2005) 
[13] de Hoog F.R., Anderssen R.S., Simple and accurate formulas for flow-curve recovery from Couette rheometer data, Applied Rheol. 16 321-328 (2006)

[14] Steffe J.F., Rheological methods in food engineering process, Second Edition, Freeman Press, East Lansing, USA, 1996

[15] Barnes H.A., Hutton J.F., Walters K., An introduction to rheology, Elsevier Science Publishing Company Inc., New-York, 1989

[16] Whorlow R.W., Rheological techniques, Second Edition, Ellis Horwood, New-York, 1992

[17] Martinez-Padilla, L.P., Rivera-Vargas, C., Flow behavior of Mexican sauces using a vane-in-a-large cup rheometer, J. Food Engineering 72 189-196 (2006)

[18] Joye D.D., Shear rate and viscosity corrections for a Casson fluid in cylindrical (Couette) geometries, J. Colloid Interface Science 267 204-210 (2003)

[19] Kelessidis V.C., Maglione R., Modeling rheological behaviour of bentonite suspensions as Casson and Robertson-Stiff fluids using Newtonian and true shear rates in Couette viscometry, Powder Tech. 168 134-147 (2006) 


\section{Figure captions}

Figure 1. Errors in calculated shear rate of Power Law fluid using different shear rate approximation techniques for various radius ratios (a) 1.04 ; (b) 2 ; (c) 3 .

Figure 2. Errors in calculated shear rate of Herschel-Bulkley fluid using different shear rate approximation techniques for various radius ratios (a) 1.04 ; (b) 2 ; (c) 3 .

Figure 3. Errors in calculated shear rate of Casson fluid using different shear rate approximation techniques for various radius ratios (a) 1.04 ; (b) 2 ; (c) 3 .

Figure 4. Comparison of the theoretical and computed Couette synthetic noisy Casson data.

Figure 5. Comparison of Couette shear flow curves of tomato ketchup computed from Steffe data, Newtonian and Bingham approximations.

Figure 6. Comparison of Couette shear flow curves of bentonite suspension computed with true shear rate from Casson model, Newtonian and Bingham approximations - (a) bentonite suspension W2 ; (b) bentonite suspension W4.

\section{Table Captions}

Table 1: geometrical and rheological parameters used to generate synthetic Couette rheometer data

Table 2: Rotational velocity vs wall shear stress for aqueous bentonite suspensions [19] 


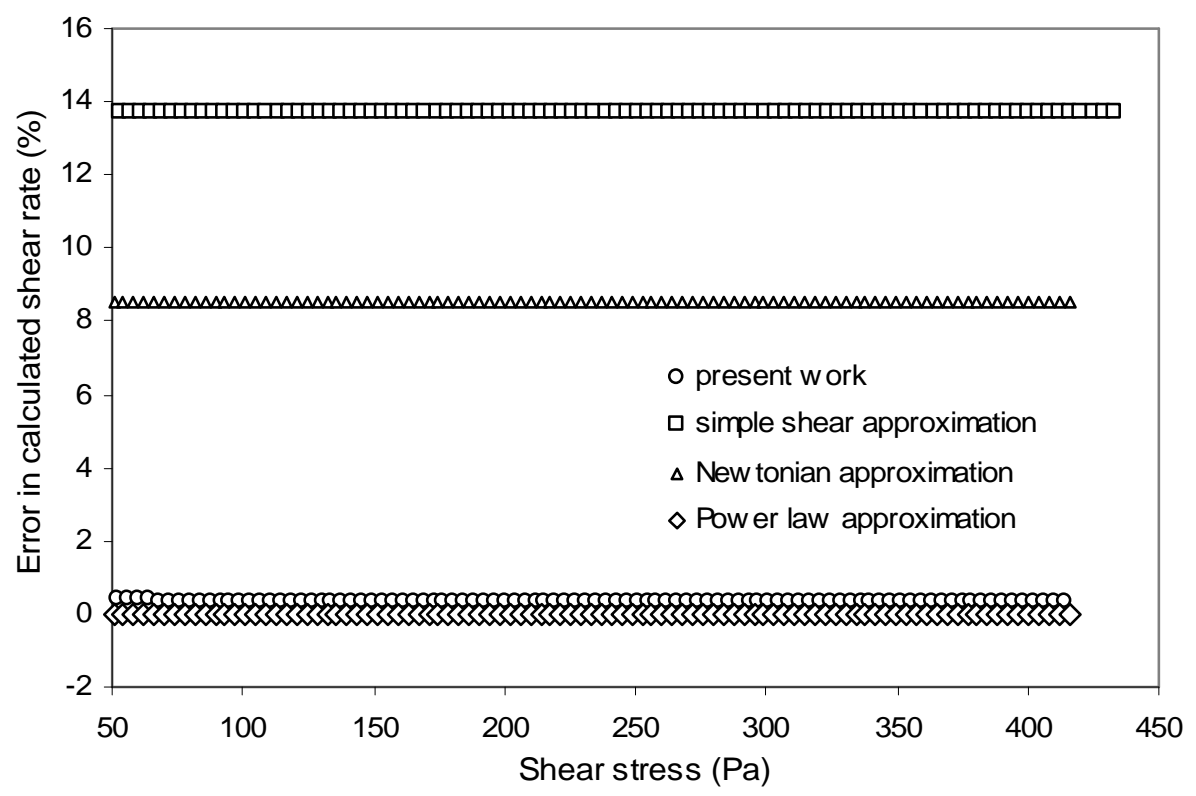

(a)

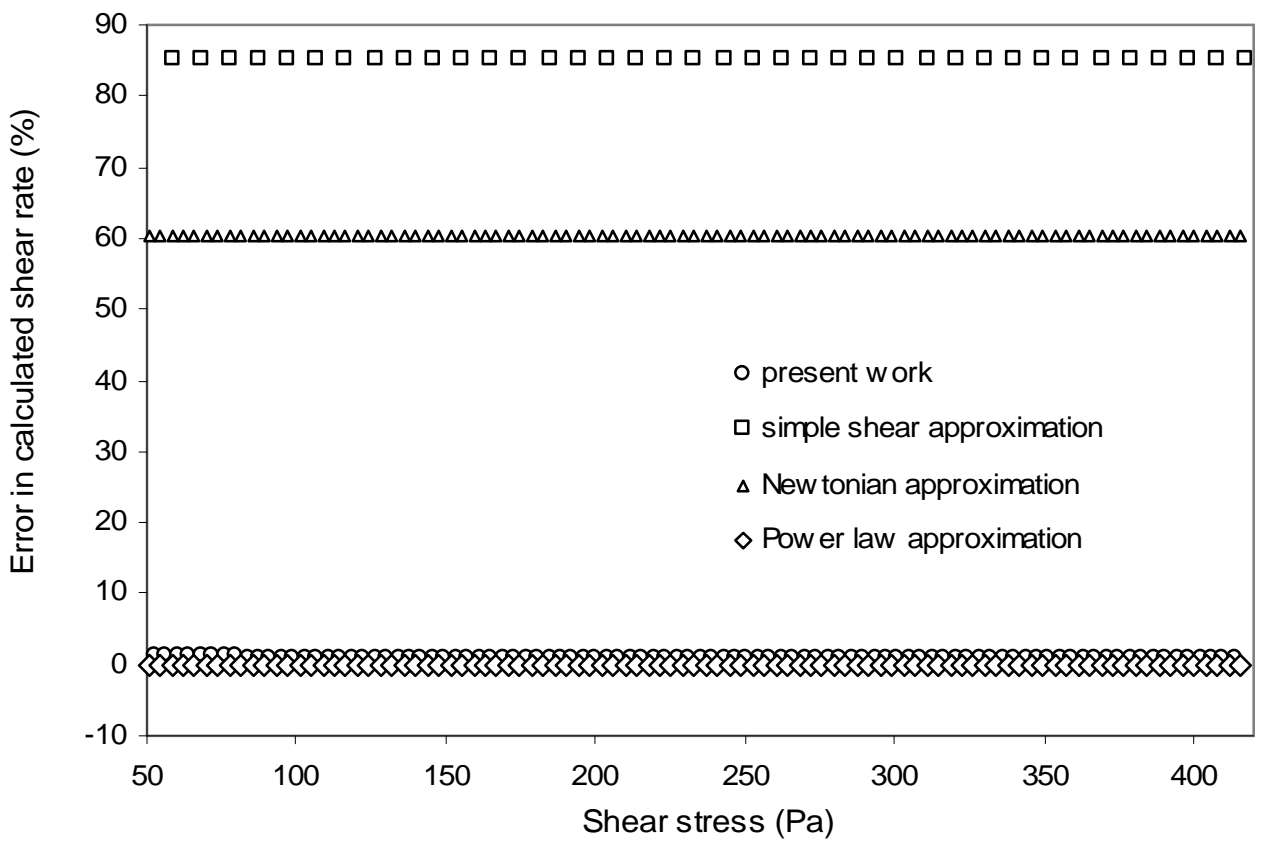

(b) 


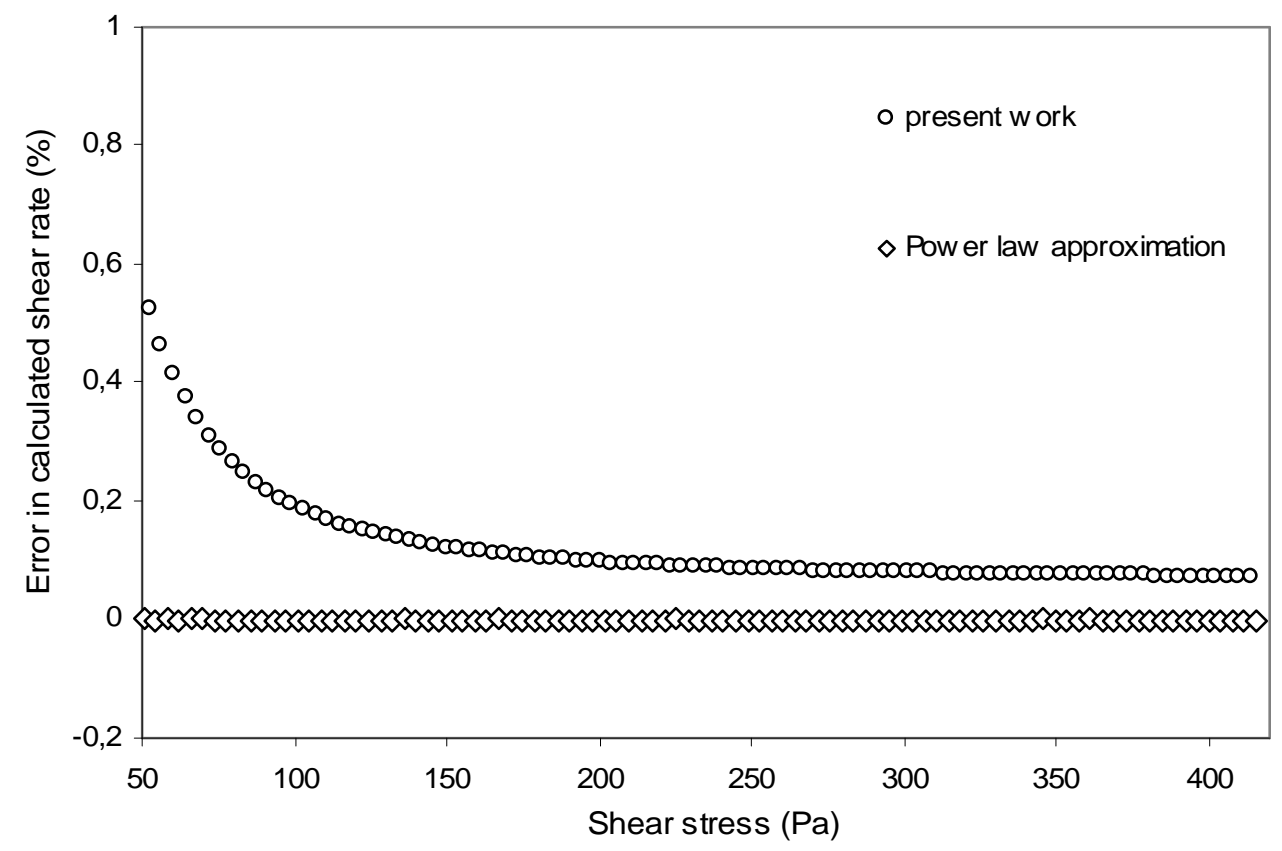

(c)

Figure 1. Errors in calculated shear rate of Power Law fluid $\left(\eta=50 \mathrm{~Pa}_{\mathrm{s}}^{\mathrm{n}} ; \mathrm{n}=0.3\right)$ using different shear rate approximation techniques for various radius ratios (a) 1.04 ; (b) 2 ; (c) 3 . 


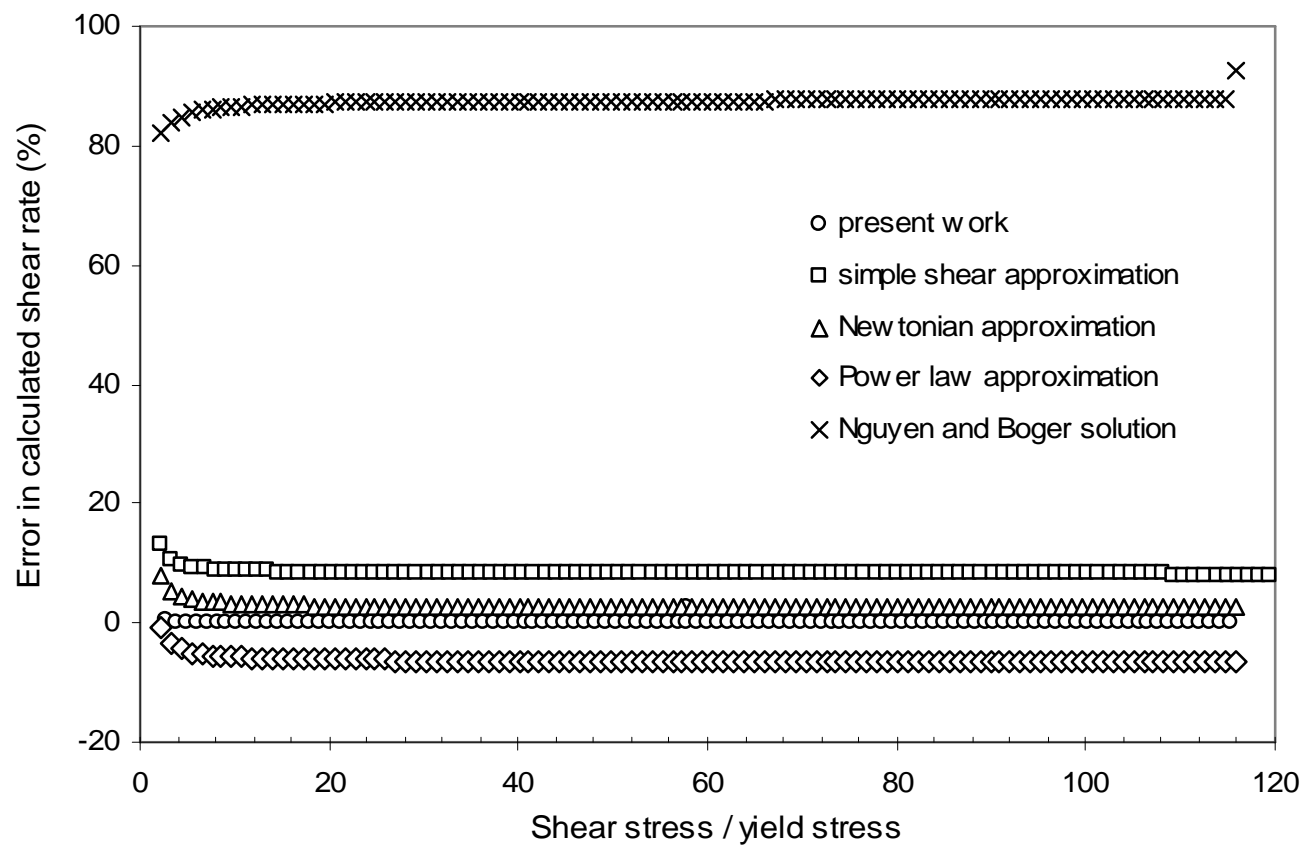

(a)

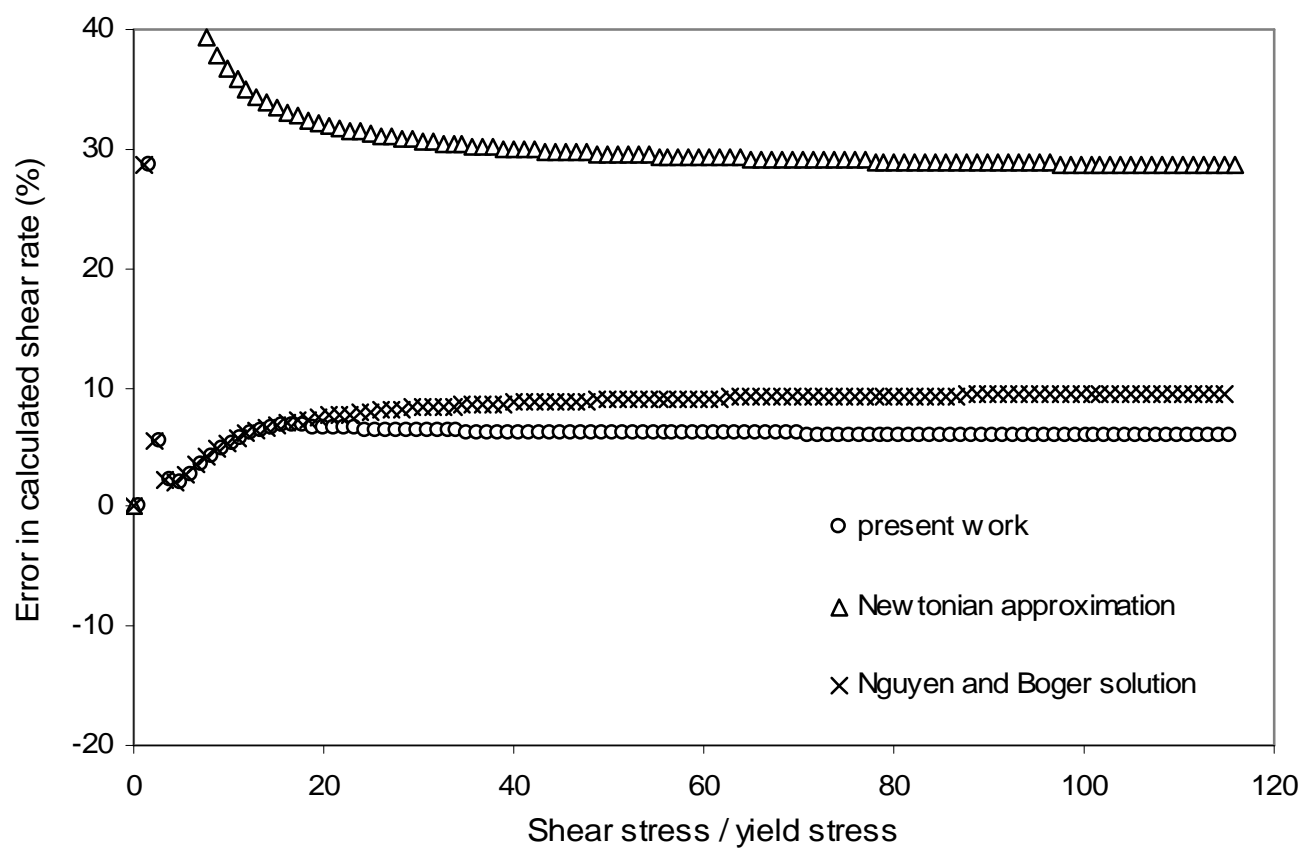

(b) 


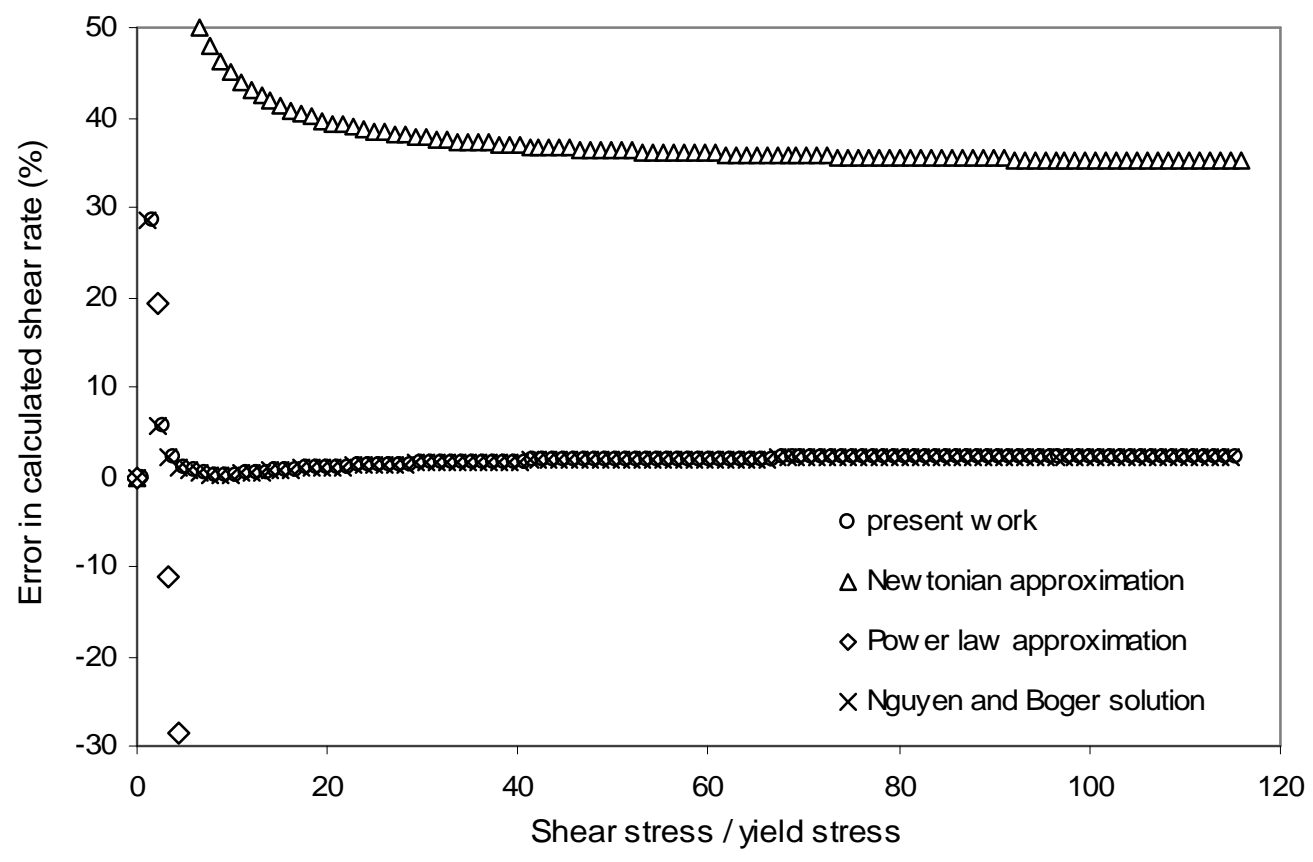

(c)

Figure 2. Errors in calculated shear rate of Herschel-Bulkley fluid using different shear rate approximation techniques for various radius ratios (a) 1.04 ; (b) 2 ; (c) 3 . 


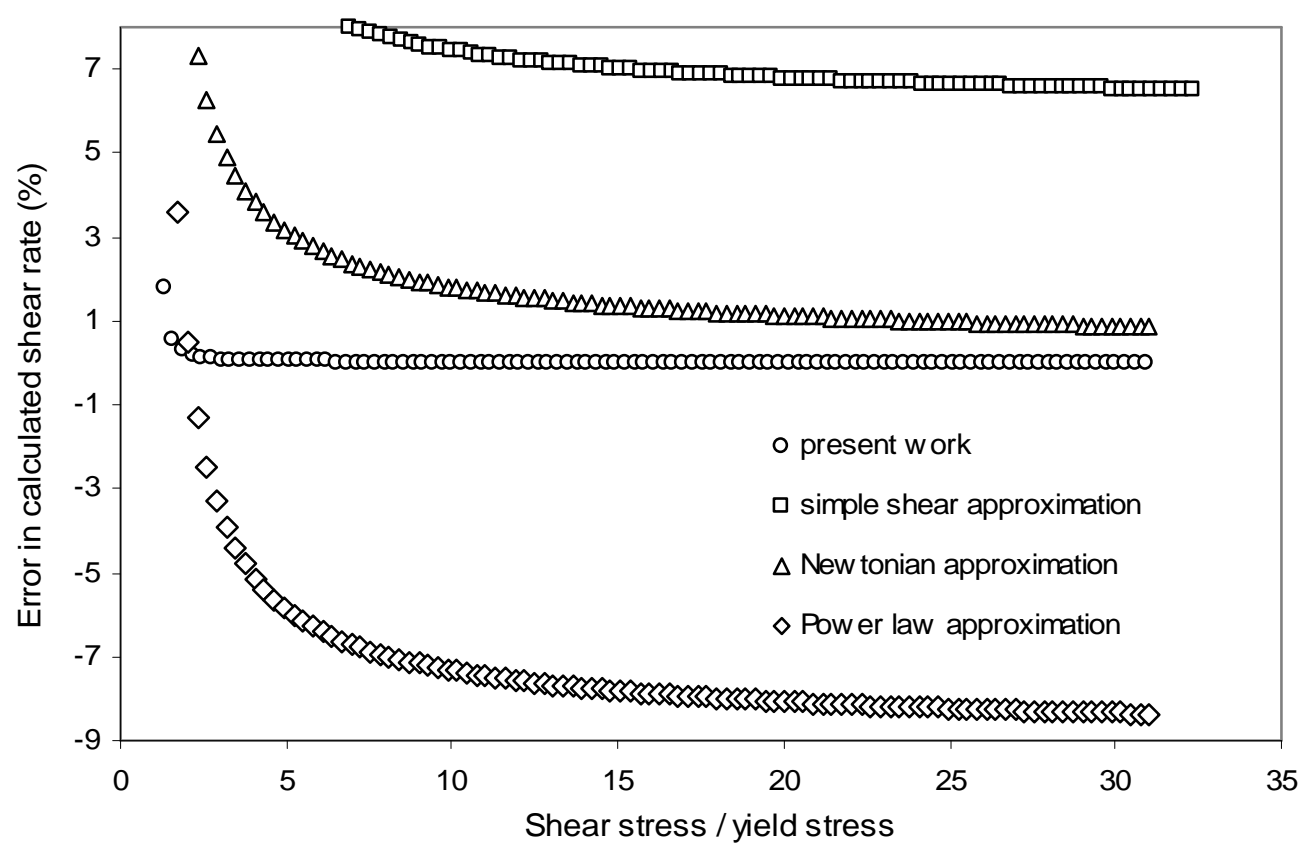

(a)

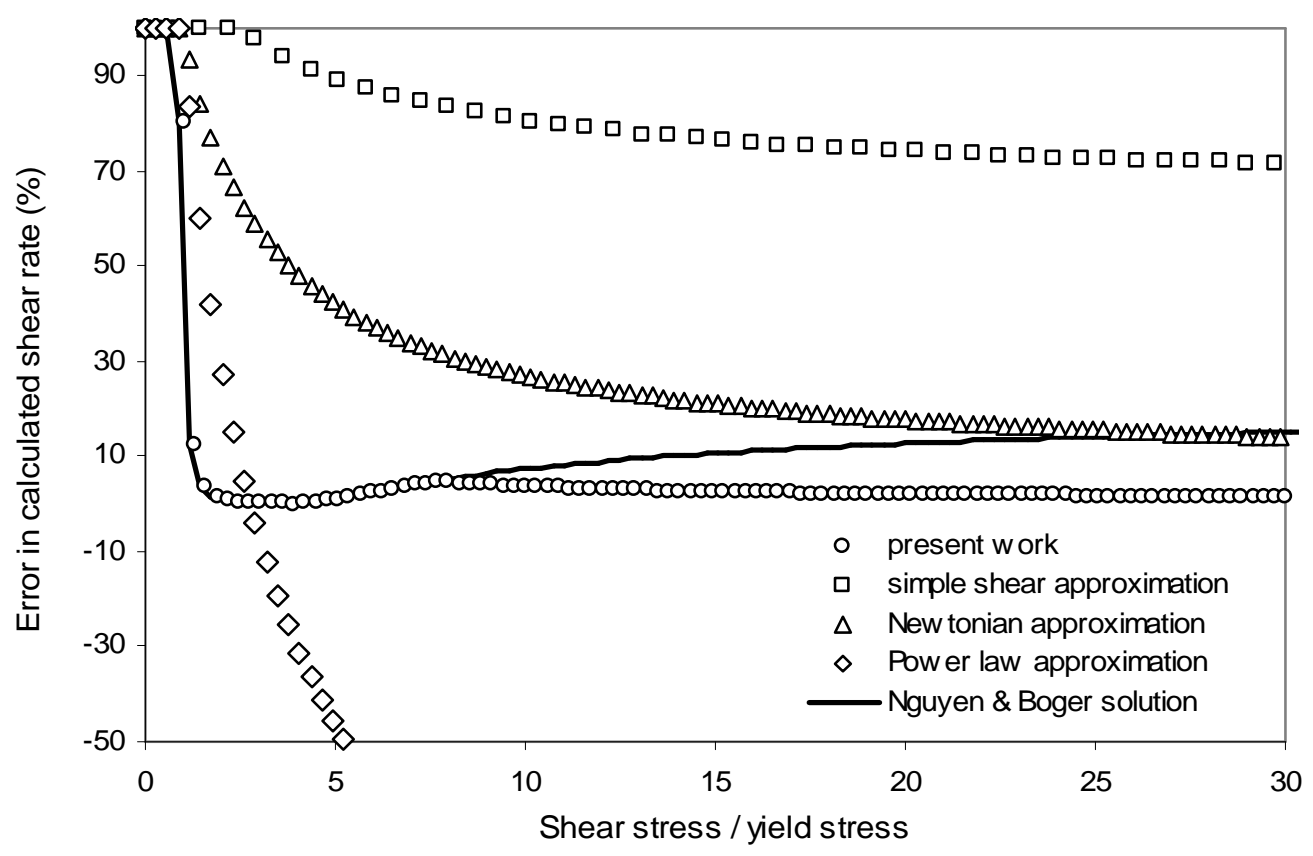

(b) 


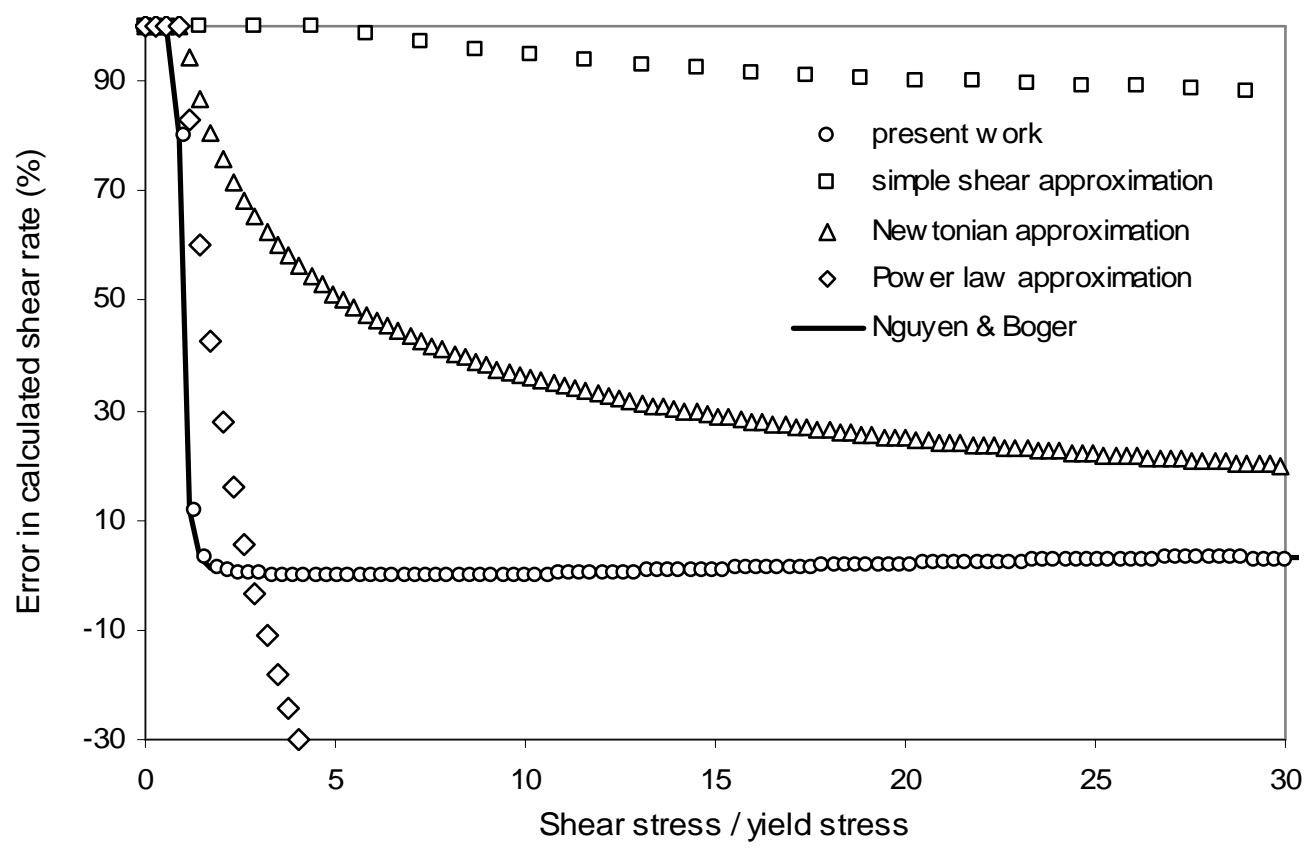

(c)

Figure 3. Errors in calculated shear rate of Casson fluid using different shear rate approximation techniques for various radius ratios (a) 1.04 ; (b) 2 ; (c) 3 . 


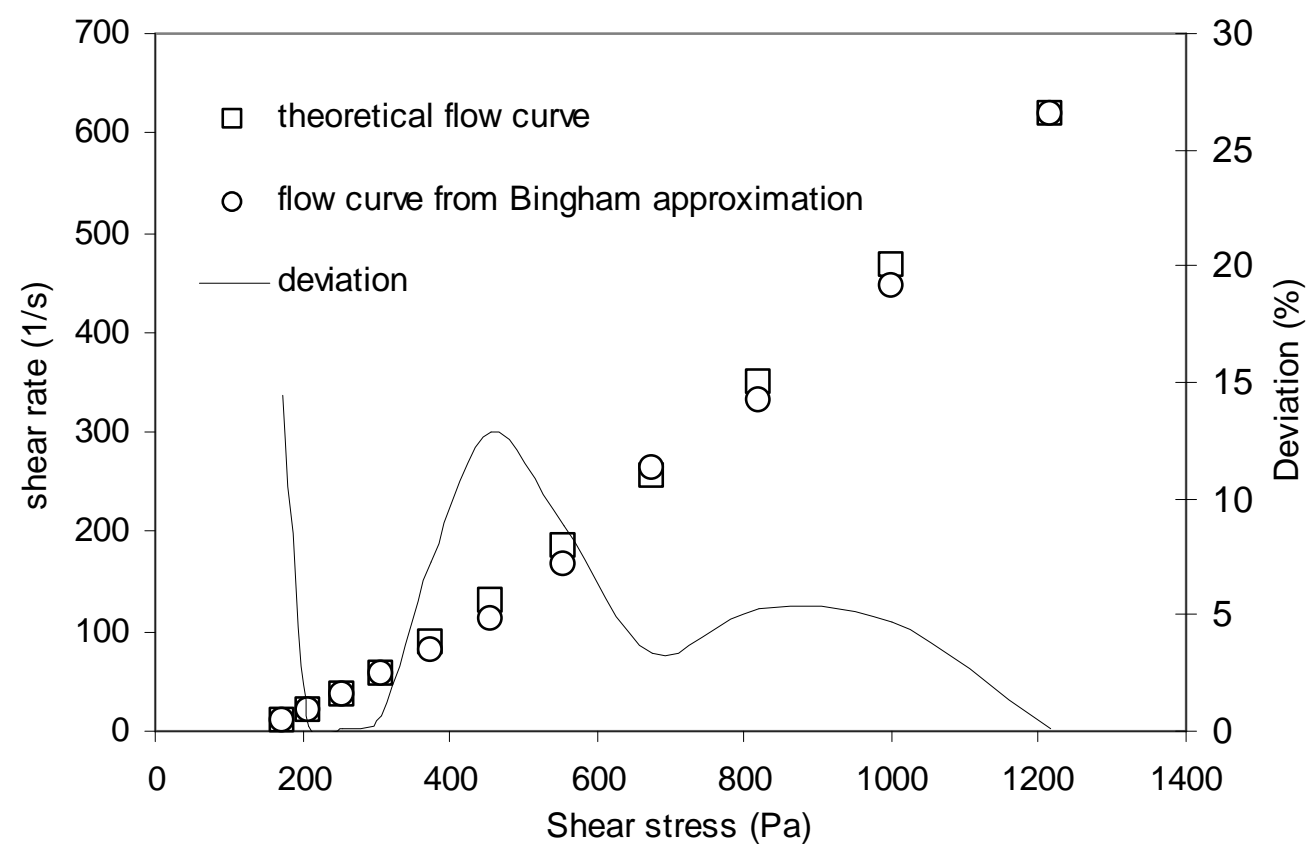

Figure 4. Comparison of the theoretical and computed Couette synthetic noisy Casson data. 


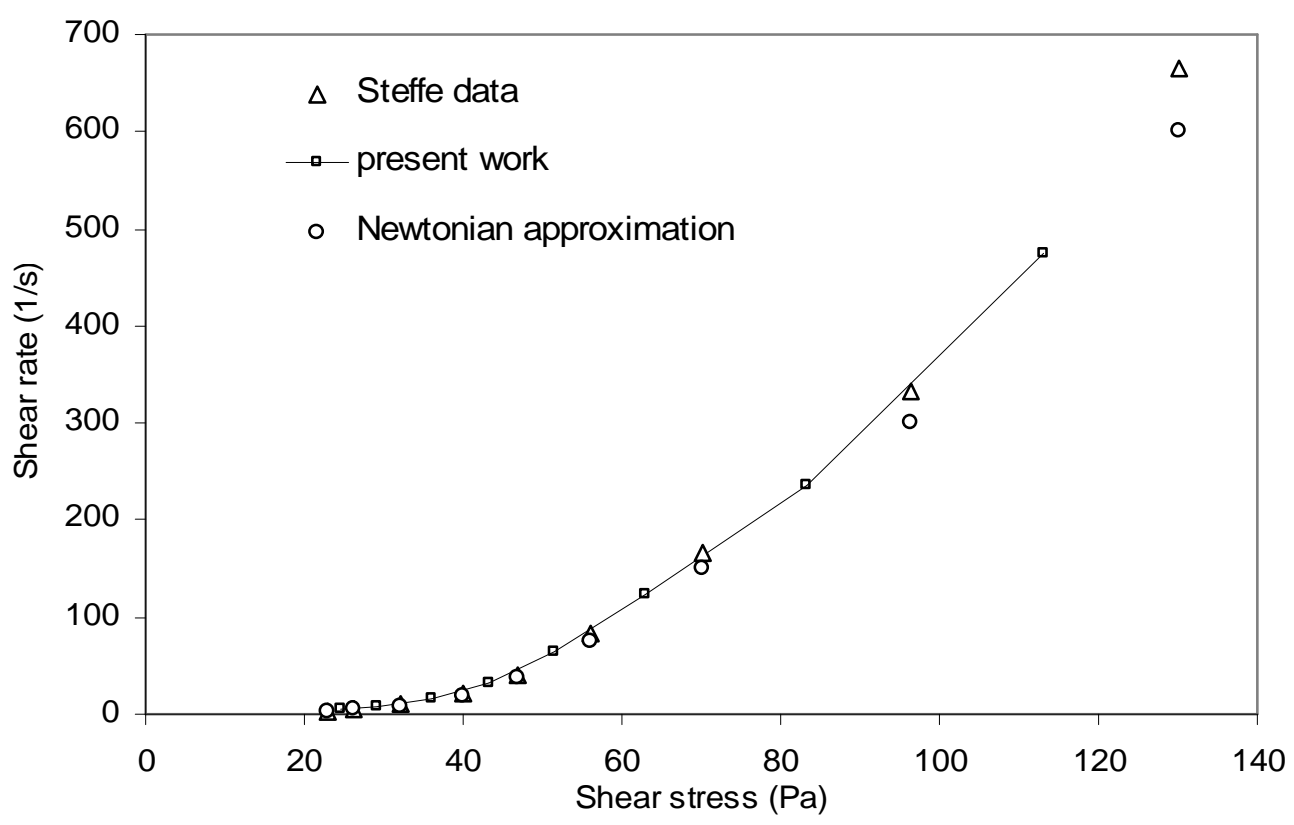

Figure 5. Comparison of Couette shear flow curves of tomato ketchup computed from Steffe data, Newtonian and Bingham approximations. 


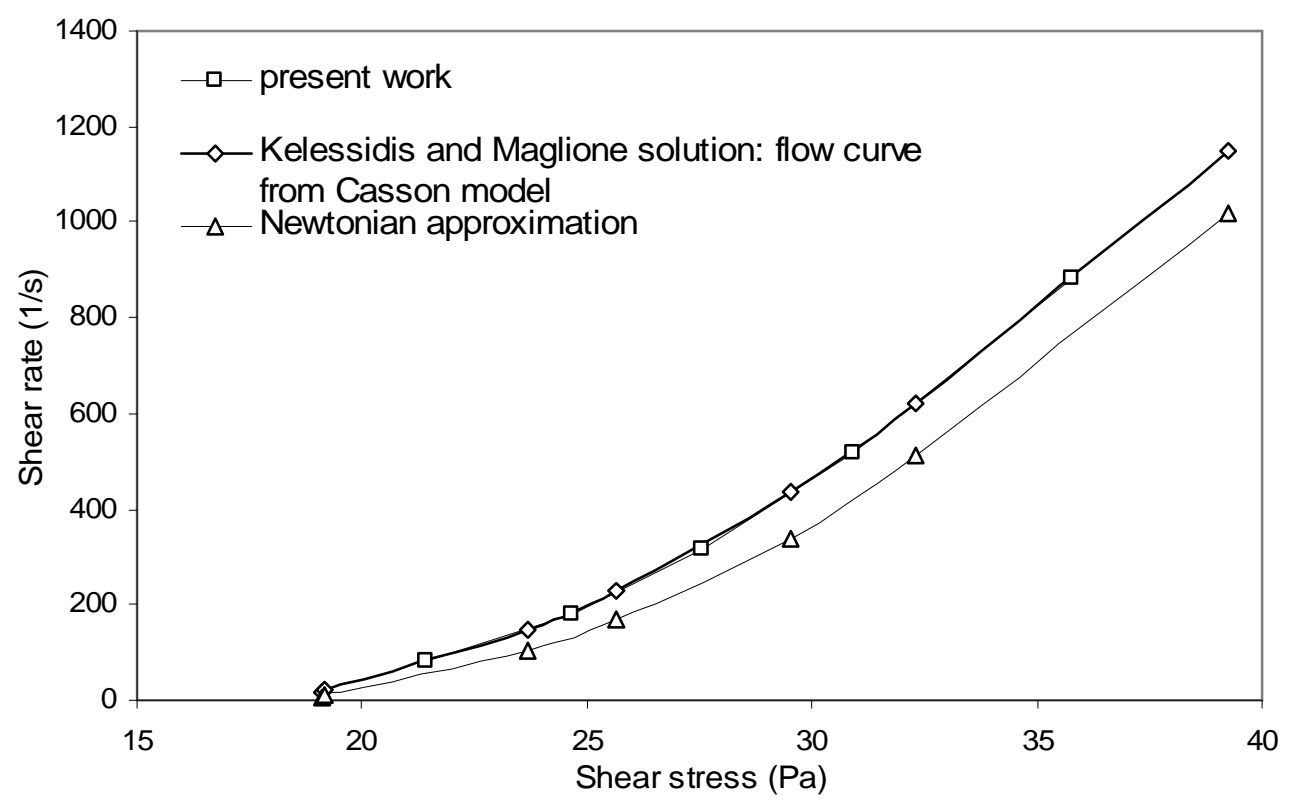

(a)

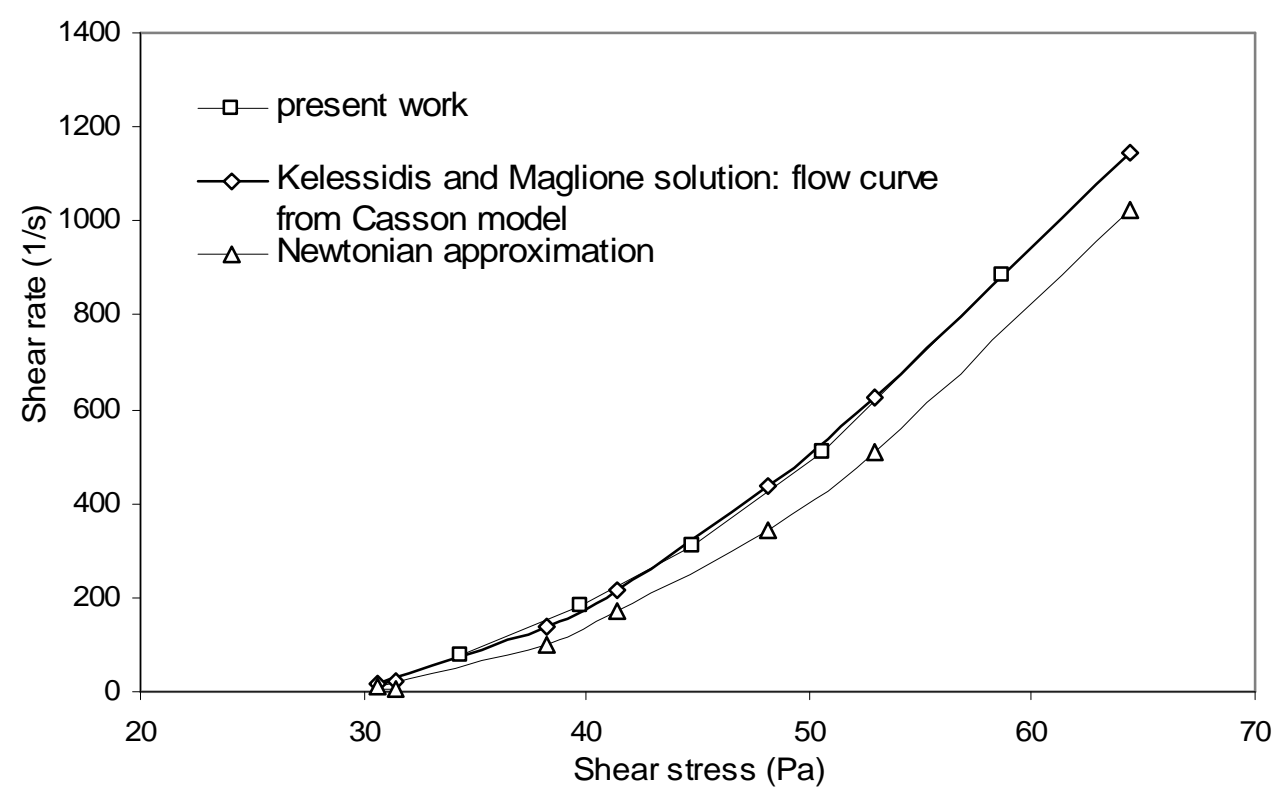

(b)

Figure 6. Comparison of Couette shear flow curves of bentonite suspension computed with true shear rate from Casson model, Newtonian and Bingham approximations - (a) bentonite suspension W2 ; (b) bentonite suspension W4. 
Table 1: geometrical and rheological parameters used to generate synthetic Couette rheometer data

\begin{tabular}{|l|c|c|c|c|c|c|}
\hline Fluid & $\mathrm{R}_{\mathrm{i}}(\mathrm{mm})$ & $\mathrm{h}(\mathrm{mm})$ & Step in torque $(\mathrm{N} . \mathrm{m})$ & $\mathrm{K}(\mathrm{Pa})$ & $\mu(\mathrm{Pa} . \mathrm{s})-\eta\left(\mathrm{Pa} . \mathrm{s}^{\mathrm{n}}\right)$ & $\mathrm{n}(-)$ \\
\hline Newtonian & 16 & 16 & 0.00046 & - & 100 & - \\
\hline Power law & 16 & 16 & 0.0001 & - & 50 & 0.3 \\
\hline Herschel-Bulkley & 14 & 20 & 0.008 & 300 & 50 & 0.6 \\
\hline Casson & 16 & 16 & 0.0005 & 100 & 1 & - \\
\hline
\end{tabular}

Table 2: Rotational velocity vs wall shear stress for aqueous bentonite suspensions [19]

\begin{tabular}{|c|c|c|}
\hline Rotational velocity $(\mathrm{rpm})$ & $\tau_{\mathrm{b}}(\mathrm{Pa})-$ suspension W2 & $\tau_{\mathrm{b}}(\mathrm{Pa})-$ suspension W4 \\
\hline 600 & 39.25 & 64.42 \\
\hline 300 & 32.33 & 53 \\
\hline 200 & 29.5 & 48.17 \\
\hline 100 & 25.67 & 41.33 \\
\hline 60 & 23.67 & 38.25 \\
\hline 6 & 19.17 & 30.58 \\
\hline 3 & 19.08 & 31.42 \\
\hline
\end{tabular}

